\title{
Complex Genetic Contribution of the Apo AI-CIII-AIV Gene Cluster to Familial Combined Hyperlipidemia

\author{
Identification of Different Susceptibility Haplotypes
}

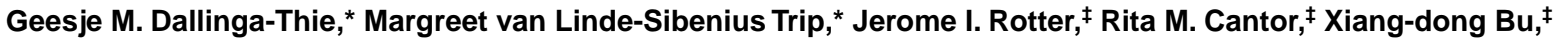 \\ Aldons J. Lusis, ${ }^{\S}$ and Tjerk W.A. de Bruin* \\ *Department of Medicine and Endocrinology, University Hospital, 3508 GA Utrecht, The Netherlands; ${ }^{\ddagger}$ Division of Medical Genetics, \\ Department of Medicine, and Department of Pediatrics, Cedars-Sinai Research Institute and University of California, Los Angeles, Los \\ Angeles, California 90048; and ${ }^{\S}$ Department of Medicine, Department of Microbiology and Molecular Genetics, and Molecular Biology \\ Institute, University of California, Los Angeles, Los Angeles, California 90024
}

\begin{abstract}
Familial combined hyperlipidemia $(\mathrm{FCH})$ is a common genetic lipid disorder in Western societies. In a recent report (Dallinga-Thie, G.M., X.D. Bu, M. van Linde-Sibenius Trip, J.I. Rotter, A.J. Lusis, and T.W.A. de Bruin. J. Lipid Res., 1996, 36:136-147) we have studied three restriction enzyme polymorphisms: XmnI, and MspI sites 5' of the apo AI gene and SstI site in the $3^{\prime}$ untranslated region of exon 4 of the apo CIII gene in $18 \mathrm{FCH}$ pedigrees, including 18 probands, 178 hyperlipidemic relatives, 210 normolipidemic relatives, and 176 spouses. DNA variations in the apo AI-CIII-AIV gene cluster had a modifying effect on plasma triglycerides, LDL cholesterol, and apolipoprotein CIII levels. In this study, combinations of haplotypes were analyzed to further characterize their interactions and effect on the expression of severe hyperlipidemia in FCH subjects. A specific combination of haplotypes with one chromosome carrying the X1M1S2 (1-1-2) haplotype and the other the X2M2S1 haplotype (2-2-1) was significantly more frequent in hyperlipidemic relatives $(6 \%)$ than in normolipidemic relatives $(3 \%)$ and spouses $(0.5 \%)$. Associated with this combination of haplotypes were significantly elevated plasma cholesterol $(P<$ 0.0001), triglycerides $(P<0.0001)$, and apo CIII $(P<0.001)$ levels when compared to the wild type combination of haplotypes 1-1-1/1-1-1. The only spouse with this specific combination of haplotypes showed a severe hyperlipidemic phenotype, similar to FCH. Furthermore, nonparametric sibpair linkage analysis revealed significant linkage between these markers in the gene cluster and the FCH phenotype (MspI $P=0.0088$, SstI $P=0.044$, and XMS haplotype $P=0.037$ ). The present findings confirm that the apo AI-CIII-IV gene cluster contributes to the FCH phenotype,
\end{abstract}

T.W.A. de Bruin's present address is Department of Medicine, Maastricht University Hospital, P.O. Box 5800, 6202 A2, Maastrict, The Netherlands.

Address correspondence to Dr. G.M. Dallinga-Thie, Departments of Medicine and Endocrinology, G 02.228, University Hospital Utrecht, P.O. Box 85500, 3508 GA Utrecht, The Netherlands. Phone: 31-30-2507355; FAX: 31-30-2518328; E-mail: g.m.dallingathie@ digd.azu.nl

Received for publication 8 August 1996 and accepted in revised form 21 December 1996.

J. Clin. Invest.

(C) The American Society for Clinical Investigation, Inc.

0021-9738/97/03/0953/09 \$2.00

Volume 99, Number 5, March 1997, 953-961 but this contribution is genetically complex. An epistatic interaction between different haplotypes of the gene cluster was demonstrated. The $\mathrm{S} 2$ allele on one haplotype was synergistic to the $\mathrm{X} 2 \mathrm{M} 2$ allele on the other haplotype in its hyperlipidemic effect. Therefore, two different susceptibility loci exist in the gene cluster, demonstrating the paradigm of complex genetic contribution to FCH. (J. Clin. Invest. 1997. 99:953-961.) Key words: familial combined hyperlipidemia • genetic linkage analysis $\cdot$ apolipoprotein $\mathrm{B} \cdot$ apolipoprotein CIII • gene-gene interaction

\section{Introduction}

Familial combined hyperlipidemia $(\mathrm{FCH})^{1}$ was described in 1973 by Goldstein et al. (1) as a distinctive genetic lipid disorder resulting in dramatically elevated plasma triglyceride levels and a secondary effect on plasma cholesterol levels. The prevalence of the disease in Western societies is about $1-2 \%$ of the population, and in survivors of myocardial infarction under the age of $60 \mathrm{yr}$ it is $\sim 10 \%$ (1), indicating a 10 -fold increased risk for myocardial infarction (2). Subsequent genetic analyses indicated that the inheritance pattern was more complex and non-Mendelian with a major gene acting on triglyceride levels (3-5). The locus with the greatest evidence for involvement is that of the apo AI-CIII-AIV gene complex on chromosome 11, but even the results with this have been confusing, controversial, and somewhat inconclusive (6). Herein, we provide data demonstrating a complex interaction of high risk haplotypes in this region. This, together with nonparametric linkage data, provide strong evidence for the involvement of this genetic region in $\mathrm{FCH}$, and begin to unravel the complexity of its genetic contribution.

$\mathrm{FCH}$ is characterized by a variable expression of both hypercholesterolemia and hypertriglyceridemia, also known as multiple type hyperlipidemia, in both probands and hyperlipidemic relatives $(2,7,8)$. Lipoprotein metabolism is characterized by an overproduction of hepatic apo B100 containing lipoproteins, resulting in increased plasma levels of VLDL and LDL (9-11). Small dense LDL particles (atherogenic lipoprotein phenotype B) are frequently found in FCH (12-14), indicating that defective regulation of apo $\mathrm{B}$ metabolism may be important in the development of FCH.

The genetic defects underlying FCH are yet unknown. Because $\mathrm{FCH}$ is associated with elevated plasma levels of apo B, cholesterol, and triglycerides, genetic factors influencing these traits may be candidate genes for the development of $\mathrm{FCH}$.

1. Abbreviation used in this paper: $\mathrm{FCH}$, familial combined hyperlipidemia. 
Despite the observation that plasma apo B levels are elevated, no associations were observed between markers near the apo $\mathrm{B}$ gene and the $\mathrm{FCH}$ phenotype $(15,16)$. Another candidate is the gene coding for the enzyme lipoprotein lipase (LPL) that is involved in the catabolism of trigyceride-rich lipoprotein particles. In FCH, a decreased LPL activity has been found in $30 \%$ of the affected individuals (17-19) that suggests that heterozygosity for LPL mutations might contribute to the lipid phenotype in FCH. However, mutations of the LPL gene (19-22) appear to contribute to only a small fraction of $\mathrm{FCH}$ and by sibpair analysis we have excluded LPL as a major cause of FCH (Lusis, A.J., G.M. Dallinga-Thie, J.I. Rotter, and T.W.A. de Bruin, manuscript in preparation).

Another candidate is the gene region of the apo AI-CIIIAIV gene cluster located on chromosome 11. In 1991 Wojciechowski et al. (23) reported linkage between FCH (analyzed as a single disorder) and this cluster, but this finding has not been confirmed (24). One possible explanation for these variant findings is that this gene cluster does contribute to the FCH phenotype but in a genetically complex manner. The apolipoproteins encoded by this cluster are implicated in the metabolism of HDL and triglyceride-rich lipoprotein particles. Several associations between polymorphisms in this cluster and parameters of lipid metabolism have been reported (2529 ) in patient groups and the general population. In a large study using 18 well-characterized FCH kindreds, we evaluated three polymorphisms in the apo AI-CIII-AIV gene cluster and their associations with lipid and apolipoprotein phenotypes were characterized (6). These findings strongly suggested that the apo AI-CIII-AIV gene cluster is not the primary cause of $\mathrm{FCH}$ but that this cluster has a specific modifying effect on plasma triglyceride and LDL cholesterol levels. In the present study a more detailed analysis of the combination of haplotypes of this gene cluster was performed. The aim was to delineate the haplotypes involved, to test whether particular combinations of haplotypes are more frequent in $\mathrm{FCH}$ individuals, and, if so, whether they result in a more severe lipid phenotype. We also performed nonparametric linkage analysis to confirm the importance of the locus in $\mathrm{FCH}$. The present data indicated that two different susceptibility loci for $\mathrm{FCH}$ in this gene cluster were present, with an S2-bearing haplotype acting in a dominant fashion, and an X2M2 haplotype behaving as a permissive trait. Furthermore, these two haplotypes exhibited epistatic interaction leading to susceptibility to FCH.

\section{Materials}

Index subjects. 18 unrelated, Dutch Caucasian, index FCH patients were recruited from the Lipid Clinic of the University Hospital, Utrecht, The Netherlands. These subjects met the criteria described previously $(1,7,30)$, including: (a) A primary hyperlipidemia with varying phenotypic expression, including a fasting plasma cholesterol concentration $>6.5 \mathrm{mmol} /$ liter or $>95$ th percentile for age, defined according to tables from the Lipid Research Clinics, and/or fasting plasma triglyceride concentration $>2.3 \mathrm{mmol} / \mathrm{liter}$, and elevated plasma apo $\mathrm{B}$ concentrations exceeding the mean +2 standard deviation for age adjusted levels. (b) At least one first-degree relative with a different hyperlipidemic phenotype from the proband. (c) A positive family history of premature coronary artery disease, defined as myocardial infarction or cerebrovascular disease before the age of $60 \mathrm{yr}$ in at least one blood-related subject or the index patient. (d) Absence of xanthomas. Exclusion criteria included diabetes, familial hypercholesterolemia (absence of isolated elevated plasma LDL cholesterol levels and tendon xanthomas), and type III hyperlipidemia (apo E2/ E2 genotype). All subjects gave informed consent. The study protocol was approved by the Human Investigation Review Committee of the University Hospital Utrecht, The Netherlands. An attempt was made to collect all relatives and spouses of the index patients, without any selection. In total, $95 \%$ of the living relatives over $18 \mathrm{yr}$ old (including 107 first degree -, 110 second degree -, and 171 third-degree relatives) have been included in the present analysis. Hyperlipidemic relatives $(n=178)$ were assigned the $\mathrm{FCH}$ phenotype when they met the following criteria: plasma cholesterol levels $>6.5 \mathrm{mmol} / \mathrm{liter}$ and/ or plasma triglycerides $>2.3 \mathrm{mmol} / \mathrm{liter}$. As a consequence, there were 210 normolipidemic relatives. The spouse group $(n=176)$ represented an environment-, nutrition-, and age-matched control group for the relatives; 58 spouses ( 25 men and 33 women) were hyperlipidemic according to the criteria described.

Analytical methods. Venous blood was drawn after an overnight fast of 12-14 h and abstention from alcohol use for at least $48 \mathrm{~h}$. Plasma was prepared by immediate centrifugation for analytical analysis. Lipids and apolipoproteins were quantified by methods as described elsewhere $(6,30,31)$.

DNA genotyping. DNA was isolated from $10 \mathrm{ml}$ of EDTA-augmented blood following standard procedures (32) and amplified by the PCR technique in a Thermal cycler apparatus (Pharmacia, Uppsala, Sweden) as described extensively (6). Three polymorphic markers, XmnI (C-2500T), MspI (G-78A), both at the 5' site of the apo AI gene, and SstI (G3175C) in the $3^{\prime}$ untranslated region of the apo CIII gene cluster were typed (6). Alleles were defined as 1 or 2 based on absence or presence of the restriction site.

Statistical methods. Results are expressed as mean \pm SD. The statistical differences between the plasma parameters of the groups were calculated using the unpaired Student's $t$ test. Data for plasma triglyceride, apo CIII, cholesterol, and apo B were analyzed unadjusted and after $\log$ transformation. Frequencies of the three polymorphisms were determined by gene counting and allele counting. Deviations of the Hardy Weinberg equilibrium were tested with a $\chi^{2}$ goodnessof-fit test. Linkage disequilibrium between the tested markers was tested using the EHDOS program (33). Haplotypes and combinations of haplotypes were assigned by examining the cosegregation of individual alleles according to Mendelian inheritance within the 18 families. Allele and haplotype frequency distributions were assessed by chi-square tests.

Nonparametric sibpair linkage analysis. The methodology of robust sibpair analysis was used to test the hypothesis that there is linkage between $\mathrm{FCH}$ as a qualitative trait and polymorphic markers in the apo AI-CIII-AIV gene cluster $(34,35)$. The basis for this approach is to compare the presence or absence of the FCH trait between siblings as a function of the alleles they share identicalby-descent (IBD). The sibpair IBD method does not require a priori assumptions about the mode of inheritance, an advantage in the analysis of genetically complex multigenic disease. In this method, the proportion of genes IBD between the members of each pair of sibs is estimated for each of the marker loci. The expected frequency of the shared alleles between affected sibs is 0.50 , when linkage to FCH is not present. A distortion from this frequency is expected when linkage to $\mathrm{FCH}$, as a qualitative trait, is present. The actual sibpair linkage analyses were performed by the SIBPAL program (SAGE version 2.4) (35). In this analysis, FCH was defined as: plasma cholesterol $>90 \%$ level for age, and/or plasma triglyceride $>2.3$ $\mathrm{mmol} / \mathrm{liter}$, and/or plasma apo B above 75 th percentile for age. Because a specific locus hypothesis was being tested, a $P$ value of 0.05 or less was considered to be significant.

\section{Results}

Subject characteristics. Clinical and biochemical characteristics of the hyperlipidemic FCH relatives including the 18 probands, their normolipidemic relatives, and spouse controls are 


\begin{tabular}{lccc}
\hline \multicolumn{1}{c}{ Variable } & Hyperlipidemic individuals* & Normolipidemic individuals & Spouses \\
\hline No. & 196 & 210 & 176 \\
Age (y) & $47 \pm 15^{\ddagger}$ & $32 \pm 13$ & $48 \pm 15^{\S}$ \\
Gender (M/F) & $115 / 81^{\text {ฯा }}$ & $105 / 105^{\text {Il }}$ & $68 / 109$ \\
BMI, kg/m & $26.20 \pm 3.55^{\ddagger}$ & $23.97 \pm 6.92$ & $25.18 \pm 3.77^{\|}$ \\
Waist-hip ratio (WHR) & $0.88 \pm 0.09^{\ddagger}$ & $0.81 \pm 0.08$ & $0.84 \pm 0.10$ \\
Chol (mmol/liter) & $7.38 \pm 2.15^{\ddagger}$ & $4.94 \pm 0.80$ & $5.66 \pm 1.05^{\S}$ \\
LDL-Chol (mmol/liter) & $4.73 \pm 1.32^{\ddagger}$ & $3.13 \pm 0.71$ & $3.67 \pm 0.98^{\S}$ \\
HDL-Chol (mmol/liter) & $1.13 \pm 0.33^{\ddagger}$ & $1.25 \pm 0.31$ & $1.28 \pm 0.36$ \\
TG (mmol/liter) & $3.29 \pm 4.92^{\ddagger}$ & $1.23 \pm 0.39$ & $1.63 \pm 1.11^{\S}$ \\
Apo B (mg/100 ml) & $130 \pm 29^{\ddagger}$ & $82 \pm 21$ & $99 \pm 28^{\S}$ \\
Apo AI (mg/100 ml) & $133 \pm 27$ & $133 \pm 23$ & $138 \pm 23^{\sharp}$ \\
Apo CIII (mg/100 ml) & $12.1 \pm 4.8^{\ddagger}$ & $7.6 \pm 2.4$ & $9.0 \pm 3.3^{\S}$ \\
& & & \\
\hline
\end{tabular}

Values are expressed as mean \pm SD. $P$ values were determined using the $t$ test. * This includes the 18 probands and 178 hyperlipidemic relatives (total number, $n=196) .{ }^{*}$ Hyperlipidemic relatives and normolipidemic relatives and spouses $P<0.001 ;{ }^{8}$ normolipidemic relatives and spouses, $P<0.001$; $\|$ normolipidemic relatives and spouses, $P<0.03$; " hyperlipidemic relatives $\left(\chi^{2}=11.1, P<0.0009\right)$, normolipidemic relatives $\left(\chi^{2}=4.8, P<0.03\right)$ versus spouses.

summarized in Table I. The group of hyperlipidemic relatives and probands was characterized by an increased BMI compared to their normolipidemic relatives and spouses. Additional differences also included an increased waist-hip ratio (WHR) and an enrichment in number of male subjects. Normolipidemic relatives were significantly younger than hyperlipidemic relatives and spouses. It cannot be excluded that in some normolipidemic individuals the $\mathrm{FCH}$ phenotype had not yet been fully expressed. Hyperlipidemic family members had significantly $(P<0.0001)$ higher plasma cholesterol, triglycerides, LDL cholesterol, apo B, and apo CIII concentrations than normolipidemic relatives and spouses. HDL cholesterol levels were significantly lower $(P<0.0001)$ compared to both normolipidemic relatives and spouses. No difference was observed in plasma apo AI levels between hyperlipidemic and normolipidemic relatives; however, in spouses the plasma apo AI levels were significantly $(P<0.03)$ higher than in normolipidemic relatives.

Polymorphisms of the apo AI-CIII-AIV gene cluster. In this study three restriction sites were studied: XmnI located $2.5-\mathrm{kb}$ upstream of the start of the apo AI gene, MspI at -78 bp from the transcription start of the apo AI gene, and SstI located in the noncoding region of exon 4 of the apo CIII gene. The observed frequencies for all three polymorphisms behaved within the limits of the Hardy Weinberg law. No differences in the gene frequencies between males and females were observed and this allowed us to combine data from men and women. The MspI locus and the XmnI locus were in strong linkage disequilibrium (6), such that those subjects who carried the X2 allele invariably had the M2 minor allele. The frequency of the M2 allele was higher than that of the X2 allele because 21 subjects carried a M2 allele but no X2 allele.

The genotype frequencies of the minor alleles of XmnI $(P<0.05)$, MspI $(P<0.05)$, and SstI $(P<0.025)$ loci were higher in hyperlipidemic relatives and probands than in spouses (Fig. 1). The difference in genotype frequency in the normolipidemic relatives versus the spouses only reached significance for MspI $(P<0.05)$, although a similar magnitude of effect was seen for XmnI. Further haplotype analysis, by specific assignment of the alleles from the coinheritance in the 18 families, showed that from the eight theoretically possible combinations of alleles only five were observed in the present study population (Table II). The potential haplotypes 2-1-2 (XmnI, MspI, SstI), 1-2-2, and 2-2-2 were not observed, indicating that the S2 minor allele never combined with the X2 and M2 alleles on one chromosome in this sample. The frequency of the common wild type haplotype (1-1-1) showed a gradual reduction from 0.795 in spouses to 0.744 in normolipidemic relatives down to 0.666 in hyperlipidemic relatives $(P<$ $0.05)$. This reduced occurrence of the common haplotype in FCH subjects was caused by a specific, significant increase of the $2-2-1(P<0.05), 1-2-1(P<0.05)$, and $1-1-2(P<0.01)$ haplotypes. The 2-2-1 haplotype also had a significantly higher frequency in normolipidemic relatives $(P<0.05)$ than in spouses.

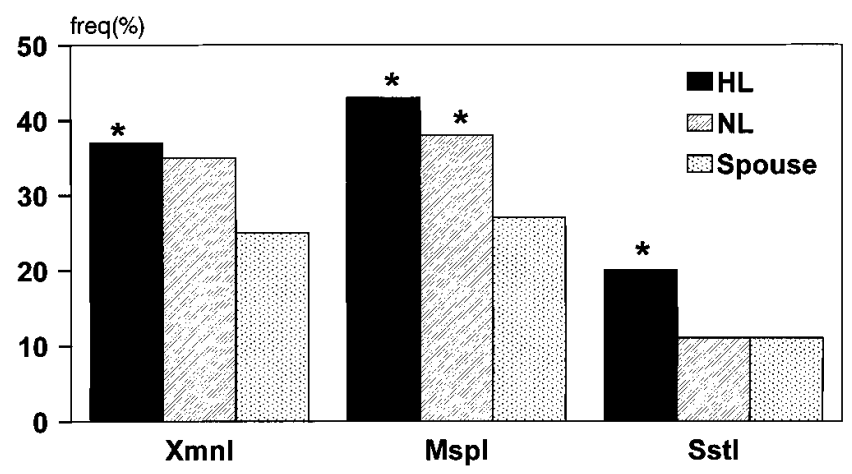

Figure 1. Relative frequencies of the minor alleles of XmnI, MspI, and SstI restriction enzyme polymorphisms. HL, hyperlipidemic relatives $(n=178)$ and probands $(n=18)$. NL, normolipidemic relatives $(n=210)$ and spouses $(n=176)$. $P$ values were determined using $\chi^{2}$-test. XmnI: HL relatives versus spouses $P<0.025$. MspI: HL relatives versus spouses $P<0.01$, NL relatives versus spouses $P<0.05$. SstI: HL relatives versus spouses $P<0.05$. 
Table II. Frequencies of Haplotypes in the FCH Population

\begin{tabular}{|c|c|c|c|}
\hline $\begin{array}{c}\text { Haplotypes } \\
\text { X-M-S* }\end{array}$ & Hyperlipidemic relatives & Normolipidemic relatives & Spouses \\
\hline & $n=392$ & $n=420$ & $n=352$ \\
\hline $1-1-1$ & $0.666^{\ddagger} \|$ & 0.744 & 0.795 \\
\hline $2-1-1$ & 0.006 & 0.003 & 0.000 \\
\hline $1-2-1$ & $0.038^{\ddagger}$ & 0.014 & 0.020 \\
\hline $1-1-2$ & $0.106^{\S \|}$ & 0.058 & 0.062 \\
\hline $2-2-1$ & $0.184^{\text {혜 }}$ & $0.181^{\mathbb{I}}$ & 0.124 \\
\hline $2-2-2$ & 0.000 & 0.000 & 0.000 \\
\hline
\end{tabular}

$P$ values were tested using Chi-square test. *X, XmnI, M, MspI, S, SstI. ${ }^{\ddagger}$ Hyperlipidemic and normolipidemic relatives $P<0.05 ;{ }^{\S}$ hyperlipidemic and normolipidemic relatives $P<0.01$; "hyperlipidemic relatives and spouses $P<0.05$; ${ }^{\mathrm{I}}$ normolipidemic relatives and spouses $P<0.05$.

In order to associate phenotypic variations with genotype variation, we analyzed the effect of the combination of haplotypes, because each individual has two copies of chromosome 11. Table III summarizes all the combination of haplotypes that were assigned by inheritance in the present $\mathrm{FCH}$ family population. Again, a gradual decline in frequency of the wild type combination of haplotypes (i.e., the homozygous 1-1-1/ 1-1-1 haplotype combination) was observed, with a gradient from 0.62 in spouses, to 0.55 in normolipidemic relatives, and down to 0.42 in hyperlipidemic relatives $(P<0.05)$. This decrease in wild type frequency was accompanied by an increased frequency of the $1-1-1 / 1-2-1$ and $1-1-1 / 2-2-1$ combination of haplotypes in both hyperlipidemic and normolipidemic relatives compared to the spouses. However, the most dramatic and only significant difference $(P<0.01)$ was the increase in frequency of the specific combination of haplotypes $1-1-2 / 2-2-1$ (Table III). In the group of spouses, representing the normal population, only 1 of 176 individuals expressed this specific combination of haplotypes, compared to 7 individuals in the group of normolipidemic relatives, and 12 individuals in the group of hyperlipidemic relatives and probands. It must be emphasized that this specific combination of haplotypes (1-1-2/

Table III. Frequency of the Different Combination of Haplotypes Observed in the FCH Population

\begin{tabular}{|c|c|c|c|c|c|c|}
\hline \multirow[t]{2}{*}{$\begin{array}{l}\text { Combined haplotype } \\
\text { X-M-S/X-M-S }\end{array}$} & \multicolumn{2}{|c|}{$\begin{array}{l}\text { Hyperlipidemic } \\
\text { relatives }\end{array}$} & \multicolumn{2}{|c|}{$\begin{array}{l}\text { Normo- } \\
\text { lipidemic } \\
\text { relatives }\end{array}$} & \multicolumn{2}{|c|}{ Spouses } \\
\hline & no. & $\%$ & no. & $\%$ & no. & $\%$ \\
\hline $1-1-1 / 1-1-1$ & 83 & $0.42 *$ & 115 & 0.55 & 110 & 0.62 \\
\hline $1-1-1 / 1-2-1$ & 11 & 0.06 & 6 & 0.03 & 3 & 0.02 \\
\hline $1-1-1 / 1-1-2$ & 24 & 0.12 & 17 & 0.08 & 17 & 0.10 \\
\hline $1-1-1 / 2-2-1$ & 59 & 0.30 & 61 & 0.30 & 40 & 0.23 \\
\hline $2-2-1 / 2-2-1$ & 0 & & 3 & 0.015 & 0 & \\
\hline $1-1-2 / 2-2-1$ & 12 & $0.06^{\ddagger}$ & 7 & 0.03 & 1 & 0.005 \\
\hline $1-1-2 / 1-1-2$ & 3 & 0.015 & 0 & & 2 & 0.01 \\
\hline $2-1-1 / 2-2-1$ & 2 & 0.010 & 1 & 0.005 & 0 & \\
\hline $1-2-1 / 2-2-1$ & 1 & 0.005 & 0 & & 2 & 0.01 \\
\hline $1-2-1 / 1-2-1$ & 1 & 0.005 & 0 & & 1 & 0.005 \\
\hline
\end{tabular}

$P$ values were tested by chi-square test. *Hyperlipidemic relatives and spouses, $P=0.041 ;{ }^{*}$ hyperlipidemic relatives and spouses, $P=0.01$.
A

B

\section{$\mathrm{Xmnl}$ \\ Mspl}

Sstl
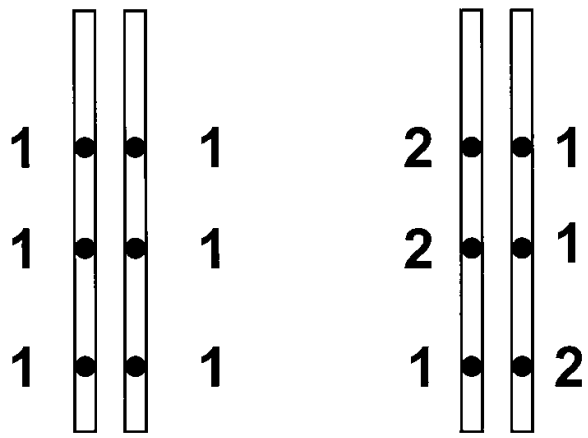

Figure 2. Schematic drawing of the common combination of haplotypes 1-1-1/1-1-1 $(A)$ and the rare combination of haplotypes $1-1-2 /$ $2-2-1(B)$, depicting the findings that $\mathrm{S} 2$ is located on a different chromosome than the $\mathrm{X} 2 \mathrm{M} 2$.

2-2-1) is characterized by the fact that the S2 allele invariably resides on a different allele than the $\mathrm{M} 2$ and $\mathrm{X} 2$ minor alleles, who are in linkage disequilibrium as shown herein and previously (6). Fig. 2 provides a schematic representation of these findings. The present analysis (Table III) revealed a six-fold increased frequency of the 1-1-2/2-2-1 combination of haplotypes in normolipidemic relatives and a 12 -fold increase in hyperlipidemic relatives. Therefore, the specific 1-1-2/2-2-1 combination of haplotypes was identified with high susceptibility for expression of the $\mathrm{FCH}$ phenotype.

Effect of combined haplotypes on quantitative phenotypes. Associations between the spectrum of combinations of haplotypes and quantitative phenotypic lipid and apolipoprotein traits in hyperlipidemic relatives, normolipidemic relatives, and spouses are summarized in Tables IV, V, and VI. Those hyperlipidemic individuals who were typed with the rare, specific high risk 1-1-2/2-2-1 combination of haplotypes had significantly higher plasma cholesterol, triglycerides $(P<0.0001)$, and apo CIII concentrations $(P<0.001)$ than the hyperlipidemic individuals with the common wild type combination of haplotypes 1-1-1/1-1-1 (Table IV, Fig. 2). In the group of spouses, only one individual was found who had the high risk combination of haplotypes. This individual had markedly elevated plasma cholesterol, LDL cholesterol, triglycerides, apo $\mathrm{B}$, and apo CIII levels, actually comparable to the levels found in the hyperlipidemic FCH group. In seven normolipidemic relatives with this specific high risk combination of haplotypes, plasma cholesterol, triglycerides, apo B, and apo CIII concentrations were higher than the normolipidemic individuals with the 1-1-1/1-1-1 combination of haplotypes, but the difference was not significant. Subsequently, an analysis was done to assess the effect of the individual haplotypes in isolation (Tables $\mathrm{V}$ and VI). The hyperlipidemic FCH individuals who had either the 1-1-1/2-2-1 (Table V) or the 1-1-1/1-1-2 (Table VI) single high risk combination of haplotypes did not show significant phenotypic variation, compared to the hyperlipidemic individuals with the wild type 1-1-1/1-1-1 combination of haplotypes, although a trend for higher plasma cholesterol, LDL cholesterol, and TG concentrations with a greater vari- 
Table IV. High Risk Combination: Effect of the High Susceptibility Risk Combination of Haplotypes X1M1S2/X2M2S1 (1-1-2/ 2-2-1) Compared with the X1M1S1/X1M1S1(1-1-1-/1-1-1) Combination of Haplotypes on Plasma Traits

\begin{tabular}{lccccc}
\hline \multicolumn{1}{c}{ Traits } & \multicolumn{2}{c}{ Hyperlipidemic relatives } & Normolipidemic relatives & Spouses \\
\hline & $1-1-1 / 1-1-1$ & $1-1-2 / 2-2-1$ & $1-1-1 / 1-1-1$ & $1-1-2 / 2-2-1$ & $1-1-1 / 1-1-1$ \\
No. & 83 & 12 & 115 & 7 & 111 \\
Chol (mmol/liter) & $7.12 \pm 1.19$ & $10.8 \pm 6.25^{*}$ & $4.86 \pm 0.85$ & $5.16 \pm 0.52$ & $5.50 \pm 1.06$ \\
LDLch (mmol/liter) & $4.72 \pm 1.31$ & $3.94 \pm 1.22^{\ddagger}$ & $3.08 \pm 0.75$ & $3.06 \pm 0.40$ & $3.54 \pm 1.00$ \\
HDLch (mmol/liter) & $1.16 \pm 0.34$ & $1.08 \pm 1.45$ & $1.23 \pm 0.28$ & $1.43 \pm 0.24$ & $1.27 \pm 0.38$ \\
TG (mmol/liter) & $2.62 \pm 1.78$ & $12.7 \pm 16.7^{*}$ & $1.22 \pm 0.39$ & $1.31 \pm 0.38$ & $1.54 \pm 0.88$ \\
Apo B (mg/dl) & $129 \pm 28$ & $140 \pm 12$ & $80 \pm 21$ & $82 \pm 2$ & $96 \pm 28$ \\
Apo AI (mg/dl) & $133 \pm 25$ & $142 \pm 37$ & $131 \pm 21$ & $147 \pm 17$ & $136 \pm 23$ \\
Apo CIII (mg/dl) & $11.9 \pm 4.5$ & $17.8 \pm 9.8^{\S}$ & $7.4 \pm 2.4$ & $8.34 \pm 1.72$ & $8.6 \pm 3.2$ \\
& & & & & 1.36 \\
\hline
\end{tabular}

$P$ values were determined using the $t$ test. Values are expressed as mean \pm SD. * Significant difference at $P<0.0001$; ${ }^{\ddagger}$ significant difference at $P<$ $0.05 ;{ }^{\S}$ significant difference at $P<0.001$.

ability (SD) was observed. Spouses with the 1-1-1/1-1-2 combination of haplotypes, with only a single high risk haplotype, were characterized by a significantly increased plasma apo CIII concentration (25\%, Table VI), reflecting the previously described effect of the S2-allele (6).

Individuals expressing the homozygous 2-2-1 (X2M2) haplotype were found only in the normolipidemic group $(n=3)$ and no specific phenotypic variations were found (data not shown). In contrast, individuals who carried two copies of the 1-1-2/1-1-2 (S2S2) were present only in the hyperlipidemic group $(n=3)$ and in spouses $(n=2)$. A trend for higher plasma cholesterol $(10 \%)$, TG $(50 \%)$, and apo CIII $(25 \%)$ concentrations was observed, but this was not statistically significant (Table VII).

Nonparametric sibpair linkage analysis. A robust nonparametric sibpair analysis method of Haseman and Elston (34) was performed on a substantial number of pairs for each polymorphic marker (Table VIII) against the presence of the FCH phenotype as a qualitative trait. The underlying basis for this approach is to compare the qualitative variation in a trait between siblings as a function of marker alleles they share identical by descent. No mode of inheritance is assumed. As can be seen, there was an excess sharing, greater than $50 \%$, of either individual alleles of the three loci within the apo AI-CIII-AIV gene cluster, as well as with the various combinations of haplotypes (XMS). The data represent evidence for linkage of the apo AI-CIII-AIV gene cluster (MspI $[P=0.0088]$, SstI $[P=$ $0.044]$, and the XMS haplotype $[P=0.037])$ to the presence of the FCH phenotype.

\section{Discussion}

Individuals diagnosed with $\mathrm{FCH}$ have a substantial increased risk of myocardial infarction under the age of $60 \mathrm{yr}$. It is, therefore, an important challenge to further elucidate the genetic defects underlying FCH. Previous association studies and one linkage study have suggested that variations of the apo AICIII-AIV gene cluster contribute importantly to $\mathrm{FCH}(6,23$, $25)$ but other studies have disputed this conclusion $(24,36)$. A recent, large association study using three polymorphisms in the apo AI-CIII-AIV gene cluster in a set of FCH families indicated that variations in this cluster modify the plasma concentrations of cholesterol and triglycerides as well as apo B and apo CIII levels in both normolipidemic and hyperlipidemic individuals (6). The results reported herein of the nonparametric linkage analysis in the same set of families indicated that the region of the apo AI-CIII-AIV gene complex showed linkage to the FCH phenotype. However, the genetic contribution of the gene cluster was found to be complex. Analysis of inherited haplotypes within the families showed that the rare allele of the SstI polymorphism (i.e., S2) was invariably located on a different haplotype than the rare alleles

Table V. Single Haplotype X2M2: Effect of the Single High Risk Haplotype Combination X1M1S1/X2M2S1 (1-1-1/2-2-1) on Plasma Traits Compared with the Wild Type X1M1S1/X1M1S1 (1-1-1/1-1-1)

\begin{tabular}{|c|c|c|c|c|c|c|}
\hline \multirow[t]{2}{*}{ Traits } & \multicolumn{2}{|c|}{ Hyperlipidemic relatives } & \multicolumn{2}{|c|}{ Normolipidemic relatives } & \multicolumn{2}{|c|}{ Spouses } \\
\hline & $1-1-1 / 1-1-1$ & $1-1-1 / 2-2-1$ & $1-1-1 / 1-1-1$ & $1-1-1 / 2-2-1$ & $1-1-1 / 1-1-1$ & $1-1-1 / 2-2-1$ \\
\hline No. & 83 & 59 & 112 & 61 & 111 & 40 \\
\hline Chol (mmol/liter) & $7.12 \pm 1.19$ & $7.52 \pm 2.82$ & $4.86 \pm 0.85$ & $5.06 \pm 0.78$ & $5.51 \pm 1.07$ & $5.88 \pm 0.97$ \\
\hline LDLch (mmol/liter) & $4.72 \pm 1.31$ & $4.82 \pm 1.44$ & $3.08 \pm 0.75$ & $3.27 \pm 0.54$ & $3.55 \pm 1.01$ & $3.83 \pm 0.84$ \\
\hline HDLch (mmol/liter) & $1.16 \pm 0.34$ & $1.18 \pm 0.32$ & $1.23 \pm 0.28$ & $1.27 \pm 0.38$ & $1.26 \pm 0.38$ & $1.33 \pm 0.33$ \\
\hline TG (mmol/liter) & $2.62 \pm 1.78$ & $3.89 \pm 10.2$ & $1.22 \pm 0.39$ & $1.20 \pm 0.36$ & $1.55 \pm 0.87$ & $1.62 \pm 0.82$ \\
\hline Apo B (mg/100 ml) & $129 \pm 28$ & $131 \pm 25$ & $80 \pm 21$ & $86 \pm 12$ & $97 \pm 19$ & $102 \pm 15$ \\
\hline Apo AI (mg/100 ml) & $133 \pm 25$ & $137 \pm 27$ & $132 \pm 21$ & $131 \pm 26$ & $135 \pm 23$ & $145 \pm 25 *$ \\
\hline Apo CIII (mg/100 ml) & $11.9 \pm 4.5$ & $12.5 \pm 8.1$ & $7.4 \pm 2.4$ & $7.9 \pm 2.5$ & $8.6 \pm 3.2$ & $9.2 \pm 3.7$ \\
\hline
\end{tabular}

$P$ values were determined using the $t$ test. Values are expressed as mean \pm SD. $*$ Significant difference at $P<0.03$. 
Table VI. Single Haplotype S2: Effect of the Single High Risk Combination of Haplotypes X1M1S1/X1M1S2 (1-1-1/1-1-2) on Plasma Traits as Compared with the Wild Type X1M1S1/X1M1S1 (1-1-1/1-1-1) Combination of Haplotypes

\begin{tabular}{|c|c|c|c|c|c|c|}
\hline \multirow[t]{2}{*}{ Traits } & \multicolumn{2}{|c|}{ Hyperlipidemic relatives } & \multicolumn{2}{|c|}{ Normolipidemic relatives } & \multicolumn{2}{|c|}{ Spouses } \\
\hline & $1-1-1 / 1-1-1$ & $1-1-1 / 1-1-2$ & $1-1-1 / 1-1-1$ & $1-1-1 / 1-1-2$ & $1-1-1 / 1-1-1$ & $1-1-1 / 1-1-2$ \\
\hline No. & 83 & 24 & 112 & 17 & 111 & 17 \\
\hline Chol (mmol/liter) & $7.12 \pm 1.19$ & $7.02 \pm 1.23$ & $4.86 \pm 0.85$ & $5.01 \pm 0.61$ & $5.50 \pm 1.06$ & $6.03 \pm 0.98$ \\
\hline LDLch (mmol/liter) & $4.72 \pm 1.31$ & $4.72 \pm 1.10$ & $3.08 \pm 0.75$ & $3.27 \pm 0.54$ & $3.54 \pm 1.00$ & $3.94 \pm 0.95$ \\
\hline HDLch (mmol/liter) & $1.16 \pm 0.34$ & $1.08 \pm 0.21$ & $1.23 \pm 0.28$ & $1.20 \pm 0.26$ & $1.27 \pm 0.38$ & $1.27 \pm 0.33$ \\
\hline TG (mmol/liter) & $2.62 \pm 1.78$ & $2.61 \pm 1.18$ & $1.22 \pm 0.39$ & $1.20 \pm 0.36$ & $1.54 \pm 0.88$ & $1.72 \pm 0.90$ \\
\hline Apo B (mg/100 ml) & $129 \pm 29$ & $128 \pm 28$ & $80 \pm 21$ & $87 \pm 17$ & $96 \pm 28$ & $108 \pm 2$ \\
\hline Apo AI (mg/100 ml) & $134 \pm 25$ & $130 \pm 22$ & $131 \pm 22$ & $136 \pm 25$ & $136 \pm 23$ & $138 \pm 23$ \\
\hline Apo CIII (mg/100 ml) & $11.9 \pm 4.5$ & $12.0 \pm 4.2$ & $7.4 \pm 2.4$ & $8.0 \pm 2.2$ & $8.6 \pm 3.2$ & $10.5 \pm 2.8^{*}$ \\
\hline
\end{tabular}

$P$ values were determined using the $t$ test. Values are expressed as mean \pm SD. * Significant difference at $P<0.02$.

of the XmnI and MspI (i.e., X2 and M2) polymorphisms. A specific, high risk, combination of haplotypes in the apo AICIII-AIV gene cluster was delineated that had a 12-times higher frequency in subjects with the $\mathrm{FCH}$ phenotype than in spouses. This specific combination of haplotypes (1-1-2/2-2-1) represents the first identification of a gene region with high susceptibility of $\mathrm{FCH}$, in support of the results of the nonparametric linkage analysis. Presence of the high risk combination of haplotypes aggravated the hypercholesterolemia and hypertriglyceridemia in probands and hyperlipidemic relatives, increased their plasma concentrations of apo CIII and apo B, and was therefore associated with a more severe $\mathrm{FCH}$ phenotype.

The MspI polymorphism is located in the promoter region of the apo AI gene at -78 , whereas the $\mathrm{XmnI}$ polymorphism is located about 2,500 bp upstream from the apo AI gene. These polymorphisms are in strong linkage disequilibrium and in the hyperlipidemic subjects in the Dutch families, the frequency of both rare alleles was significantly increased (6). The MspI polymorphism has been reported to be associated with elevated plasma apo AI and HDL levels (37-39), but we were unable to confirm this association in either $\mathrm{FCH}$ relatives or the group of spouses (6). The MspI site contributed to quantitative variations in plasma concentrations of apo B-containing lipoproteins, i.e., plasma LDL cholesterol in spouses, and total apo B in $\mathrm{FCH}$ probands. The XmnI polymorphic site contributed to variations in plasma apo B concentrations in the group of spouses, and total cholesterol and TG in FCH probands (6). The SstI polymorphism is located in the noncoding region of exon 4 of the apo CIII gene. The minor S2 allele has been associated with hypertriglyceridemia (40-42) and elevated apo CIII levels, but this was not observed in all studies $(43,44)$. We previously reported that spouses who carry the S2 allele have significantly higher plasma cholesterol (10\%), triglyceride (40\%), apo B (14\%), and apo CIII (23\%) concentrations (6). Hyperlipidemic relatives from $\mathrm{FCH}$ families who carry the S2 allele show elevated plasma TG (56\%) and apo CIII $(21 \%)$ concentrations. The genotype frequency of the S2-allele was significantly higher in the group of hyperlipidemic relatives and probands only (by twofold; Fig. 1), with a lower, but similar frequency in normolipidemic relatives and spouses. In contrast, frequencies of the M2 and X2 alleles were higher in both the group of normolipidemic relatives and hyperlipidemic relatives compared to spouses.

Because of the frequency distribution of the SstI polymorphism in FCH families, the possibility emerged that the S2 minor allele was located on a different haplotype than the X2 and M2 minor alleles. This was indeed confirmed by the analysis of the observed haplotypes constructed from the three polymorphic markers by observing the cosegregation of alleles among the family members. As expected, haplotypes that contained a minor allele were more frequent in hyperlipidemic relatives or normolipidemic relatives. The minor $\mathrm{S} 2$ allele never cosegregated with $\mathrm{X} 2$ and/or M2 alleles on the same chromosome.

Table VII. S2 Homozygosity: Effect of Two Copies of the S2-Bearing Haplotype X1M1S2/X1M1S2 (1-1-2/1-1-2) on Plasma Traits as Compared with the Wild Type X1M1S1/X1M1S1 (1-1-1/1-1-1) Combination of Haplotypes

\begin{tabular}{lcccc}
\hline \multicolumn{1}{c}{ Traits } & \multicolumn{2}{c}{ Hyperlipidemic relatives } & Spouses \\
\hline & $1-1-1 / 1-1-1$ & $1-1-2 / 1-1-2$ & $1-1-1 / 1-1-1$ & $1-1-2 / 1-1-2$ \\
No. & 83 & 3 & 2 & 111 \\
Chol (mmol/liter) & $7.12 \pm 1.19$ & $7.82 \pm 1.77$ & $3.50 \pm 1.06$ & $6.69 \pm 0.45$ \\
LDLch (mmol/liter) & $4.72 \pm 1.31$ & $4.32 \pm 1.80$ & $1.54 \pm 1.00$ & $4.88 \pm 0.10$ \\
HDLch (mmol/liter) & $1.16 \pm 0.34$ & $1.07 \pm 0.34$ & $1.54 \pm 0.88$ & $1.24 \pm 0.14$ \\
TG (mmol/liter) & $2.62 \pm 1.78$ & $3.66 \pm 2.77$ & $96 \pm 28$ & $133 \pm 36$ \\
Apo B (mg/100 ml) & $129 \pm 29$ & $126 \pm 5$ & $136 \pm 23$ & $142 \pm 7$ \\
Apo AI (mg/100 ml) & $134 \pm 25$ & $139 \pm 31$ & $8.6 \pm 3.2$ & $9.7 \pm 1.8$ \\
Apo CIII (mg/100 ml) & $11.9 \pm 4.5$ & $14.0 \pm 7.6$ & & \\
\end{tabular}

Values are expressed as mean $\pm \mathrm{SD}$. 
Table VIII. Nonparametric Test for Sibpair Linkage Analysis

\begin{tabular}{lccc}
\hline Polymorphic marker & $\begin{array}{c}\text { Number of } \\
\text { affected sibpairs }\end{array}$ & $\begin{array}{c}\text { Frequency of } \\
\text { shared alleles }\end{array}$ & $P$ value \\
\hline & & $(\bar{x} \pm S D)^{*}$ & \\
XmnI & 196 & $0.517 \pm 0.18$ & 0.092 \\
MspI & 193 & $0.533 \pm 0.19$ & 0.0088 \\
SstI & 172 & $0.521 \pm 0.15$ & 0.044 \\
XMS haplotype ${ }^{\ddagger}$ & 184 & $0.533 \pm 0.25$ & 0.037 \\
\hline
\end{tabular}

Linkage between the presence of the $\mathrm{FCH}$ phenotype, as a qualitative trait, in affected sibpairs and genetic variation at the apo AI-CIII-AIV gene cluster was analyzed. The frequency of shared alleles and shared haplotypes (XMS) in affected FCH siblings identical-by-descent was tested for deviations of the normal, expected distribution (0.50). *Null hypothesis: proportion of shared alleles $=0.500 ;{ }^{*} \mathrm{XMS}$ haplotype, the various combinations of haplotypes.

Therefore, haplotypes of further interest were (X-M-S): 2-2-1, $2-1-1$, or $1-2-1$, together present in $22.8 \%$ of hyperlipidemic relatives, $19.8 \%$ of normolipidemic relatives, and in $14.4 \%$ of spouses $(P<0.01 ; 1.6$ to 1.4 times more frequent in relatives). The 2-2-1 haplotype was the most commonly found minor allele haplotype. The other haplotype of interest was the 1-1-2 combination, with a significantly increased (1.7 times) frequency in hyperlipidemic relatives compared to spouses. A specific, high risk, combination of haplotypes was then identified: one chromosome containing the M2, X2, and S1 alleles (2-2-1) and the other chromosome containing the X1, M1, and S2 allele (1-1-2). This relatively rare combination of haplotypes was 12 times more frequent in the group of hyperlipidemic FCH relatives and probands compared to spouses. The high-risk combination of haplotypes was associated with a dramatic increase in plasma cholesterol $(52 \%)$, triglycerides (485\%), and apo CIII $(50 \%)$ concentrations $(P<0.05)$. In the group of spouses $(n=176)$ only one individual was found with this combination of haplotypes, suggesting that the population frequency is $\sim 1: 200$. This latter individual was also characterized by elevated plasma cholesterol (31\%), triglycerides (sevenfold), and apo CIII (73\%) concentrations, similar to the FCH phenotype.

Interestingly, the present observations show that the two different haplotypes (2-2-1 and 1-1-2) contribute to plasma lipid traits in a different fashion (Table IX). Each of these contributing high risk haplotypes was also analyzed in isolation, i.e., in combination with the wild type haplotype (1-1-1). The effect of the S2 allele in this combination (1-1-1/1-1-2) was significant on plasma apo CIIII concentrations in the group of spouses, but not on other lipid traits, either in spouses or relatives. Five subjects who were homozygous for S2 (1-1-2/1-1-2) were found, three within the group of hyperlipidemic relatives and two in the spouse group, showing higher plasma total cholesterol, TG (not the two spouses), and apo CIII concentrations. The 1-1-2 haplotype therefore behaved like a dominant allele on certain traits that are part of the $\mathrm{FCH}$ phenotype (see Table IX). A different situation was found with the 2-2-1 haplotype. No significant effects of the 1-1-1/2-2-1 combination of haplotypes on plasma traits were found. Three individual X2M2 (2-2-1/2-2-1) homozygous subjects were identified, each belonging to the group of normolipidemic relatives. The data therefore indicate that the 2-2-1 haplotype does not act
Table IX. Model of Genetic Susceptibility to FCH Conferred by Apo AI-CIII-AIV Gene Cluster Haplotypes

\begin{tabular}{ll}
\hline \multicolumn{1}{c}{ Haplotype (XmmI-MspI-SstI) } & \multicolumn{1}{c}{$\begin{array}{c}\text { Effect on FCH } \\
\text { expression }\end{array}$} \\
\hline Wild type: $1-1-1 / 1-1-1$ & resistant \\
X2M2: $2-2-1^{*}$ & permissive \\
S2: $1-1-2^{\ddagger}$ & susceptible \\
High risk: $(\mathrm{X} 2 \mathrm{M} 2 \mathrm{~S} 1 / \mathrm{X} 1 \mathrm{M} 1 \mathrm{~S} 2): 2-2-1 / 1-1-2$ & highly susceptible
\end{tabular}

See also tables II and III, and Fig. 2 for haplotype codes. *Any combination, except $1-1-2 ;{ }^{\ddagger}$ any combination, except $2-2-1$.

in a dominant fashion, in contrast to its 1-1-2 counterpart. However, the haplotype 2-2-1 did not appear to act in a recessive fashion either, because the homozygous state was neutral with regards to its effect on lipids. The data can be interpreted that the wild type 1-1-1 haplotype is a resistance locus for hyperlipidemia, and that the 2-2-1 allele renders the locus permissive to the hyperlipidemic effect of other genes, one being the S2 allele when it occurs on the other haplotype. Such an interpretation is supported by the previous observation that the presence of any minor allele (X2, M2, S2), analyzed in isolation, resulted in a more hyperlipidemic phenotype compared to $1-1-1$ (6). In other words, the $2-2-1$ allele enhances penetrance of the hyperlipidemia and hyperlipoproteinemia associated with $\mathrm{FCH}$. This might explain the mechanism of the dramatic synergism between 2-2-1 haplotype and the 1-1-2 haplotype with regard to the expression of FCH. The data indicate that the genetic contribution to the $\mathrm{FCH}$ phenotype is complex and that at least two different and separate predisposing genetic susceptibility regions exist within the apo AI-CIIIAIV gene cluster, one characterized by the 2-2-1 allele, and the other by the 1-1-2 allele (Table IX). Both maternal and paternal chromosomes may therefore contribute to expression of the $\mathrm{FCH}$ phenotype. The data confirm a paradigm of complex genetic contribution of the apo AI-CIII-AIV gene cluster to the expression of $\mathrm{FCH}$.

The presence of multiple susceptibility regions within a gene cluster is not unprecedented, because in insulin-dependent diabetes mellitus (IDDM) a comparable phenomenon has been described (45-47). Both the DR3 and DR4 HLA linked alleles predispose to IDDM. Susceptibility for IDDM is greatly increased when they occur together as DR3/DR4 heterozygotes, compared to HLA DR3/DR3 or HLA DR4/DR4 homozygotes, the latter of which also show increased susceptibility to IDDM. The characteristic is that each of the DR3 and DR4 alleles separately contributes to susceptibility (45-47). The effect of the high risk haplotypes on the presence of $\mathrm{FCH}$, or severity of hyperlipidemia, appears to be analogous but with distinctive differences, as outlined above. The X2M2S1 allele has no effect by itself and no clear dosage effect, in contrast to the X1M1S2 allele. The more pronounced effect of the $\mathrm{S} 2$ allele on the risk to express FCH might be caused by the fact that the SstI polymorphic site is in strong linkage disequilibrium with specific mutations in the promoter region of the apo CIII gene (42). Mutations in the promoter region could affect the level of transcription of the genes in the cluster. Two polymorphisms located within an insulin responsive element (48) might be responsible for this effect. Carriers of these mutations are at increased risk for severe hypertriglyceridemia 
(42). However, two recently published studies $(49,50)$ suggested that the observed association between hypertriglyceridemia and the $\mathrm{S} 2$ allele could not be explained by linkage disequilibrium between the SstI polymorphism and variations in the insulin response element of the apo CIII gene promoter. Apo CIII is an apolipoprotein that plays a role in the elimination of remnants of TG-rich lipoproteins. We showed that impaired elimination of postprandial lipoproteins in FCH subjects is associated with increased plasma apo CIII concentrations (30). The presently identified gene variations most likely do not represent the major gene defect in $\mathrm{FCH}$, which is thought to be responsible for the hypersecretion of apo $\mathrm{B}$ containing lipoproteins. The concept that arises from this and a previous study (6) is that the apo AI-CIII-AIV gene cluster is a modifier gene, capable of increasing the penetrance of $\mathrm{FCH}$ in a susceptible individual against the background of the yet unknown major gene(s).

Nonparametric sibpair linkage analysis on the qualitative trait of $\mathrm{FCH}$ phenotype, based on age- and gender-adjusted plasma cholesterol, triglycerides, and apo B levels was used to evaluate formally the involvement of the gene cluster in $\mathrm{FCH}$. Sibpair linkage analysis $(34,35)$ is a nonparametric method that is used to compare variations in a qualitative, or quantitative, trait between siblings as a function of the marker alleles they share identical-by-descent. With complex traits such as hypertension (51) and determinants of LDL particle size (52), linked genetic loci could be identified using this methodology. In contrast, LOD score-linkage analysis (33) requires specification of a genetic model, which is difficult to establish when studying complex diseases like FCH. The current findings that MspI, and SstI polymorphic sites, as well as the haplotypes, showed significant linkage with the presence of the FCH phenotype further established the involvement of this cluster as a susceptibility gene for $\mathrm{FCH}$. The present data indicate the presence of two susceptibility alleles within or nearby this cluster that appear to be in linkage disequilibrium either with the $\mathrm{X} 2 \mathrm{M} 2$ allele or with the S2 minor allele.

In conclusion, a specific and relatively rare combination of haplotypes in the apo AI-CIII-AIV gene cluster had a 12times higher prevalence in subjects with $\mathrm{FCH}$ compared with spouses, who showed a frequency of $\sim 1$ in 200 . This high susceptibility haplotype aggravated the hypercholesterolemia, hypertriglyceridemia, and hyperlipoproteinemia in both probands and hyperlipidemic relatives. The data demonstrate the presence of two separate susceptibility alleles for $\mathrm{FCH}$ in the gene cluster, confirming a paradigm of complex genetic contribution of the apo AI-CIII-AIV gene cluster to FCH.

\section{Acknowledgments}

We thank Dr. M. Castro Cabezas for help in collecting the families. We thank the patients, relatives, and spouses for participating in this study.

This study was supported by a grant from the Dutch Heart Foundation (D 91.101), by the National Institutes of Health grant HL28481, and by the Cedars-Sinai Board of Governor's Chair in Medical Genetics (J.I. Rotter). The results of the SIBPAL analysis were obtained by using the program package SAGE, and was supported by U.S. Public Health Service Resource grant 1 P41 RR03655 from the Division of Research Resources. T.W.A. de Bruin is senior clinical investigator of the Dutch Heart Foundation and recipient of the PIONEER grant of the Dutch Organization for Fundamental Research.

\section{References}

1. Goldstein, J.L., H.G. Schrott, W.R. Hazzard, E.L. Bierman, and A.G. Motulsky. 1973. Hyperlipidemia in coronary heart disease II. Genetic analysis of lipid levels in 176 families and delineation of a new inherited disorder, combined hyperlipidemia. J. Clin. Invest. 52:1544-1568.

2. Castro Cabezas, M., T.W.A. de Bruin, and D.W. Erkelens. 1992. Familial combined hyperlipidemia: 1973-1991. Neth. J. Med. 40:83-95.

3. Iselius, L. 1981. Complex segregation analysis of hypertriglyceridemia. Hum. Hered. 31:222-226.

4. Williams, W.R., and J.M. Lalouel. 1982. Complex segregation analysis of hyperlipidemia in a Seattle sample. Hum. Hered. 32:24-36.

5. Cullen, P., B. Farren, J. Scott, and M. Farrall. 1994. Complex segregation analysis provides evidence for a major gene acting on serum triglyceride levels in 55 british families with familial combined hyperlipidemia. Arterioscler. Thromb. 14:1233-1249.

6. Dallinga-Thie, G.M., X.D. Bu, M. Van Linde-Sibenius Trip, J.I. Rotter, A.J. Lusis, and T.W.A. De Bruin. 1996. Apolipoprotein A-I/C-III/A-IV gene cluster in familial combined hyperlipidemia: Effects on LDL-cholesterol and apolipoproteins B and C-III. J. Lipid Res. 37:136-147.

7. Brunzell, J.D., J.J. Albers, A. Chait, S.M. Grundy, E. Groszek, and G.B. McDonald. 1983. Plasma lipoproteins in familial combined hyperlipidemia and monogenic familial hypertriglyceridemia. J. Lipid Res. 24:147-155.

8. Kwiterovich, P.O. 1993. Genetics and molecular biology of familial combined hyperlipidemia. Curr. Opin. Lipidol. 4:133-143.

9. Kissebah, A.H., S. Alfarsi, and P.W. Adams. 1981. Integrated regulation of very low density lipoprotein triglyceride and apolipoprotein-B kinetics in man: normolipidemic subjects, familial hypertriglyceridemia and familial combined hyperlipidemia. Metab. Clin. Exp. 30:856-868.

10. Cortner, J.A., P.M. Coates, M.J. Bennett, D.R. Cryer, and N.-A. Le. 1991. Familial combined hyperlipidemia: use of stable isotopes to demonstrate overproduction of very low density apolipoprotein B by the liver. J. Inherited Metab. Dis. 14:915-922.

11. Venkatesan, S., P. Cullen, P. Pacy, D. Halliday, and J. Scott. 1993. Stable isotopes show a direct relation between VLDL apoB overproduction and serum triglyceride levels and indicate a metabolically and biochemically coherent basis for familial combined hyperlipidemia. Arterioscler. Thromb. 13:11101118.

12. Austin, M.A., J.D. Brunzell, W.L. Fitch, and R.M. Krauss. 1990. Inheritence of low density lipoprotein subclass patterns in familial combined hyperlipidemia. Arterioscler. Thromb. 10:520-530.

13. Jarvik, G.P., J.D. Brunzell, M.A. Austin, R.M. Krauss, A.G. Motulsky, and E. Wijsman. 1994. Genetic predictors of FCHL in four large pedigrees: Influence of ApoB level major locus predicted genotype and LDL subclass phenotype. Arterioscler. Thromb. 14:1687-1694.

14. Bredie, S.J.H., L.A. Kiemeney, A.F.J. de Haan, P.N.M. Demacker, and A.F.H. Stalenhoef. 1996. Inherited susceptibility determines the distribution of dense low-density lipoprotein subfraction profiles in familial combined hyperlipidemia. Am. J. Hum. Genet. 58:812-822.

15. Rauh, G., H. Schuster, B. Muller, S. Schewe, C. Keller, G. Wolfram, and N. Zollner. 1990. Genetic evidence from seven families that the apolipoprotein $\mathrm{B}$ gene is not involved in familial combined hyperlipidemia. Atherosclerosis. 83: 81-87.

16. Coresh, J., T.H. Beaty, P.O. Kwiterovich, and S.E. Antonarakis. 1992. Pedigree and sib-pair linkage analysis suggest the apolipoprotein B gene is not the major gene influencing plasma apolipoprotein B levels. Am. J. Hum. Genet. 50:1038-1045.

17. Babirak, S.P., B.G. Brown, and J.D. Brunzell. 1992. Familial combined hyperlipidemia and abnormal lipoprotein lipase. Arterioscler. Thromb. 12: 1176-1183.

18. Nevin, D.N., J.D. Brunzell, and S.S. Deeb. 1994. The LPL gene in individuals with familial combined hyperlipidemia and decreased LPL activity. Arterioscler. Thromb. 14:869-873.

19. Gagne, E., J. Genest, H. Zhang, L.A. Clarke, and M.R. Hayden. 1994. Analysis of DNA changes in the LPL gene in patients with familial combined hyperlipidemia. Arterioscler. Thromb. 14:1250-1257.

20. Reymer, P.W.A., B.E. Groenemeyer, E. Gagne, L. Miao, E.E.G. Appelman, J.C. Seidel, D. Kromhout, S.M. Bijvoet, K. Van de Oever, T. Bruin, et al. 1995. A frequently occurring mutation in the lipoprotein lipase gene (Asn291Ser) contributes to the expression of familial combined hyperlipidemia. Hum. Mol. Genet. 4:1543-1549.

21. Hoffer, M.J.V., S.J.H. Bredie, D.I. Boomsma, P.W.A. Reymer, J.J.P. Kastelein, P. de Knijff, P.N.M. Demacker, A.F.H. Stalenhoef, L.M. Havekes, and R.R. Frants. 1996. The lipoprotein lipase (Asn291-Ser) mutation is associated with elevated lipid levels in families with familial combined hyperlipidemia. Atherosclerosis. 119:159-168.

22. Mailly, F., Y. Tugrul, P.W.A. Reymer, T. Bruin, M. Seed, B.F. Groenemeyer, A. Asplund-Carlson, D. Vallance, A.F. Winder, G.J. Miller, et al. 1995. A common variant in the gene for lipoprotein lipase (Asp9 $\rightarrow$ Asn): Functional implications and prevalence in normal and hyperlipidemic subjects. Arterioscler. Thromb. 15:468-478.

23. Wojciechowski, A.P., M. Farrall, P. Cullen, T.M.E. Wilson, J.D. Bayliss, 
B.A. Griffin, M.D. Caslake, C.J. Packard, J. Shepherd, R. Thakker, et al. 1991. Familial combined hyperlipidemia linked to the apolipoprotein AI-CIII-AIV gene cluster on chromosome 11q23-q24. Nature (Lond.). 349:161-164.

24. Wijsman, E.M., A.G. Motulsky, S.W. Guo, M. Yang, M.A. Austin, J.D. Brunzell, and S. Deeb. 1992. Evidence against linkage of familial combined hyperlipidemia to the apo AI-CIII-AIV gene complex. Circulation. 86:I 420.

25. Hayden, M.R., H. Kirk, H. Campbell, J. Frohlich, S. Rabkin, R. McLeod, and J. Hewitt. 1987. DNA polymorphism in and around the apo AICIII genes and genetic hyperlipidemia. Am. J. Hum. Genet. 40:421-430.

26. Galton, D.J. 1988. Molecular genetics of coronary heart disease. Eur. J. Clin. Invest. 18:219-225.

27. Ordovas, J.M., F. Civeira, J.R. Genest, S. Craig, A.H. Robbins, T. Meade, M. Pocovi, P.M. Frossard, U. Masharani, P.W. Wilson, et al. 1991. Restriction fragment length polymorphisms of the apolipoprotein A-I, C-III, A-IV gene locus. Relationships with lipids, apolipoproteins, and premature coronary artery disease. Atherosclerosis. 87:75-86.

28. Paulweber, B., W. Friedl, F. Krempler, S.E. Humphries, and F. Sandhofer. 1988. Genetic variation in the apolipoprotein A-IC-IIIA-IV gene cluster and coronary heart disease. Atherosclerosis. 73:125-133.

29. Tybjaerg-Hansen, A., B.G. Nordestgaard, L.U. Gerdes, O. Faergeman, and S.E. Humphries. 1993. Genetic markers in the apo AI-CIII-AIV gene cluster for combined hyperlipidemia, hypertriglyceridemia, and predisposition to atherosclerosis. Atherosclerosis. 100:157-169.

30. Castro Cabezas, M., T.W.A. de Bruin, H.A. de Valk, C.C. Shoulders, H. Jansen, and D.W. Erkelens. 1993. Impaired fatty acid metabolism in familial combined hyperlipidemia. J. Clin. Invest. 92:160-168.

31. De Bruin, T.W.A., C.B. Brouwer, J.A. Gimpel, and D.W. Erkelens. 1991. Postprandial decrease in HDL cholesterol and HDL apo A-I in normal subjects in relation to triglyceride metabolism. Am. J. Physiol. 260:492-498.

32. Miller, S.A., D.D. Dykes, and H.F. Polesky. 1988. A simple salting out procedure for extracting DNA from human nucleated cells. Nucl. Acids Res. 16: 1215.

33. Terwilliger, J.D., and J. Ott. 1994. Handbook of Human Genetic Linkage. John Hopkins University Press, Baltimore. 307 pp.

34. Hasemann, J.K., and R. C. Elston. 1992. The investigation of linkage between a quantitative trait and a marker locus. Behav. Genet. 2:3-19.

35. S.A.G.E. Statistical Analysis for Genetic Epidemiology. Version 2.4 1994. System requirement: DOS. Louisiana State University Medical Center, New Orleans, LA.

36. Xu, C.-F., P. Talmud, H. Schuster, R. Houlston, G. Miller, and S. Humphries. 1994. Association between genetic variation at the Apo AI-CIIIAIV gene cluster and familial combined hyperlipidemia. Clin. Genet. 46:385397.

37. Pagani, F., A. Sidoli, G.A. Giudici, L. Barenghi, C. Vergani, and F.E. Baralle. 1990. Human apolipoprotein A-I gene promoter polymorphism: association with hyperalphalipoproteinemia. J. Lipid Res. 31:1371-1377.

38. Talmud, P., S. Ye, and S.E. Humphries. 1994. Polymorphism in the promoter region of the apolipoprotein AI gene associated with differences in apolipoprotein AI levels: the European Atherosclerosis Research Study. Genet. Epidemiol. 11:265-280.

39. Jeenah, M., A. Kessling, N. Miller, and S. Humphries. 1990. G to A substitution in the promotor region of the apolipoprotein A-I gene is associated with elevated serum apolipoprotein A-I and high density lipoprotein cholesterol concentration. Mol. Biol. Med. 7:233-241.

40. Shoulders, C.C., P.J. Harry, L. Lagrost, S.E. White, N.F. Shah, J.D. North, M. Gilligan, P. Gambert, and M.J. Ball. 1991. Variation at the apo AI/ CIII/AIV gene complex is associated with elevated plasma levels of apo CIII. Atherosclerosis. 87:239-247.

41. Rees, A., J. Stocks, L.G. Williams, J.L. Caplin, N.I. Jowett, A.J. Camm, and D.J. Galton. 1985. DNA polymorphism in the apolipoprotein C-III and insulin gene and atherosclerosis. Atherosclerosis. 58:269-275.

42. Dammerman, M., L.A. Sandkuyl, J.J. Halaas, W. Chung, and J.L. Breslow. 1993. An apolipoprotein C-III haplotype protective against hypertriglyceridemia is specified by promotor and $3^{\prime}$ untranslated region polymorphisms. Proc. Natl. Acad. Sci. USA. 90:4562-4566.

43. Helio, T., A. Paliotie, T. Sane, M.J. Tikkanen, and K. Kontula. 1994. No evidence for linkage between familial hypertriglyceridemia and apolipoprotein B, apolipoprotein C-III or lipoprotein lipase genes. Hum. Genet. 94:271-278.

44. Paul-Hayase, H., M. Rosseneu, D. Robinson, J.P. v. Biervliet, J.P Deslypere, and S.E. Humphries. 1992. Polymorphisms in the apolipoprotein AI-CIII-AIV gene cluster: detection of genetic variation determining plasma apo AI, apo CIII and apo AIV concentrations. Hum. Genet. 88:439-446.

45. Rotter, J.I., C.E. Anderson, R. Rubin, J.E. Congleton, P.I. Terasaki, and D.L. Rimoin. 1983. HLA genotypic study of insulin-dependent diabetes. Diabetes. 32:169-174.

46. Thomson, G., W.P. Robinson, M.K. Kuhner, S. Joe, M.J. MacDonald, J.L. Goitschall, J. Barbosa, S.S. Rich, J. Bertrams, M.P. Baur, et al. 1988. Genetic heterogeneity, modes of inheritance, and risk estimates for a joint study of Caucasians with insulin-dependent diabetes mellitus. Am. J. Hum. Genet. 43: 799-816.

47. Rotter, J.I., C.M. Vadheim, and D.L. Rimoin. 1992. Diabetes mellitus. In The Genetic Basis of Common Diseases. R.A. King, J.I. Rotter, and A.G. Motulsky, editors. Oxford University Press, New York. 413-481.

48. Li, W.W., M.M. Dammerman, J.D. Smith, S. Metzger, J.L. Breslow, and T. Leff. 1995. Common genetic variation in the promoter of the human apo CIII gene abolishes regulation by insulin and may contribute to hypertriglyceridemia. J. Clin. Invest. 96:2601-2605.

49. Surguchov, A.P., G.P. Page, L. Smith, W. Patsch, and E. Boerwinkle. 1996. Polymorphic markers in apolipoprotein C-III gene flanking regions and hypertriglyceridemia. Arterioscler. Thromb. Vasc. Biol. 16:941-947.

50. Shoulders, C.C., T.T. Grantham, J.D. North, A. Gaspardone, F. Tomai, A. De Fazio, F. Versaci, P.A. Gioffre, and N.J. Cox. 1996. Hypertriglyceridemia and the apolipoprotein CIII gene locus: Lack of association with the variant insulin response element in Italian school children. Hum. Genet. 98:557-566.

51. Wu, D.-A., X. Bu, C.H. Warden, D.D.C. Shen, C.-Y. Yeng, W.H.H. Sheu, M.M.T. Fuh, T. Katsuya, V.J. Dzau, G.M. Reaven, et al. 1996. Quantitative trait locus mapping of human blood pressure to a genetic region at or near the lipoprotein lipase gene locus on chromosome 8p22. J. Clin. Invest. 97:21112118

52. Rotter, J.I., X.D. Bu, R.M. Cantor, C.H. Warden, J. Brown, R.J. Gray, P.J. Blanche, R.M. Krauss, and A.J. Lusis. 1996. Multilocus genetic determinants of LDL particle size in coronary artery disease families. Am. J. Hum. Genet. 58:585-594. 